\title{
Half-Metallic Ferromagnetism Induced by Dynamic Electron Correlations in VAs
}

\author{
L. Chioncel, ${ }^{1,2} \mathrm{Ph}$. Mavropoulos, ${ }^{3}$ M. Ležaić, ${ }^{3}$ S. Blügel, ${ }^{3}$ E. Arrigoni, ${ }^{1}$ M. I. Katsnelson, ${ }^{4}$ and A. I. Lichtenstein ${ }^{5}$ \\ ${ }^{1}$ Institute of Theoretical Physics, Graz University of Technology, A-8010 Graz, Austria \\ ${ }^{2}$ Faculty of Science, University of Oradea, RO-410087 Oradea, Romania \\ ${ }^{3}$ Institut für Festkörperforschung, Forschungscentrum Jülich, D-52425 Jülich, Germany \\ ${ }^{4}$ University of Nijmegen, NL-6525 ED Nijmegen, The Netherlands \\ ${ }^{5}$ Institute of Theoretical Physics, University of Hamburg, DE-20355 Hamburg, Germany
}

(Received 26 January 2006; published 16 May 2006)

\begin{abstract}
The electronic structure of the VAs compound in the zinc-blende structure is investigated using a combined density-functional and dynamical mean-field theory approach. Contrary to predictions of a ferromagnetic semiconducting ground state obtained by density-functional calculations, dynamical correlations induce a closing of the gap and produce a half-metallic ferromagnetic state. These results emphasize the importance of dynamic correlations in materials suitable for spintronics.
\end{abstract}

PACS numbers: 75.50.Cc, 71.10. $-\mathrm{w}, 71.20 . \mathrm{Be}, 85.75 .-\mathrm{d}$

There is a growing interest in finding potential materials with high spin polarization. Suitable candidates are halfmetallic ferromagnets (HMF) which are metals for one spin direction and semiconductors for the other [1,2]. As a result, HMF are expected to show a $100 \%$ polarization and can, in principle, conduct a fully spin-polarized current at low temperature. Therefore, HMF are ideal candidates for potential applications in spintronics $[2,3]$. These materials are equally interesting for basic research, in particular, concerning the origin of the half-metallic gap, the nature of interatomic exchange interactions [4], its stability at elevated temperatures [5], or the effect of electron correlations [2].

Most theoretical efforts for understanding HMF are supported by first-principles calculations, based on densityfunctional theory (DFT). In fact, the very discovery of HMF was due to such calculations [1]. DFT calculations are usually based on the local spin density approximation (LSDA) or the generalized gradient approximation (GGA). These approximations have been proved very successful to interpret or even predict material properties in many cases, but they fail notably in the case of strongly correlated electron systems. For such systems, the so-called LSDA + $U$ (or GGA $+U$ ) method is used to describe static correlations, whereas dynamical correlations can be approached within the LSDA + DMFT (dynamical mean-field theory) [6,7]. An important dynamical many-electron feature of half-metallic ferromagnets is the appearance of nonquasiparticle states $[2,8,9]$ which can contribute essentially to the tunneling transport in heterostructures containing HMF $[10,11]$.

Equally interesting materials for spintronics applications are ferromagnetic semiconductors [12,13]. Candidate systems are ordered compounds such as europium chalcogenides (e.g., $\mathrm{EuO}$ ) and chromium spinels (e.g., $\mathrm{CdCr}_{2} \mathrm{Se}_{4}$ ) [12], as well as diluted magnetic semiconductors (e.g., $\mathrm{Ga}_{1-x} \mathrm{Mn}_{x} \mathrm{As}$ ) [13]. Unfortunately, all of them have Curie temperatures much lower than room temperature. On the other hand, VAs in the zinc-blende structure is, according to density-functional calculations [14], a ferromagnetic semiconductor with a high Curie temperature. Unlike CrAs [15], CrSb [16], and MnAs [17], VAs has not yet been experimentally fabricated in the zinc-blende structure, but the increasing experimental activity in the field of the (structurally metastable) zinc-blende ferromagnetic compounds is promising in this respect.

In this Letter, we investigate the effect of electronic interactions on VAs in the zinc-blende structure using dynamical mean-field theory. Our main result is displayed in Fig. 2 (especially the inset): While this material is expected to be a ferromagnetic semiconductor from densityfunctional theory (LSDA or GGA) or static LSDA $+U$ calculations, the inclusion of dynamic Coulomb correlations within the LSDA + DMFT approach predicts a metallic behavior, due to the closure of the gap in the majority-spin band. Moreover, since the minority-spin band gap remains finite, the material is found to be a half-metallic ferromagnet. To our knowledge, this is a first example in which dynamic correlations transform a semiconductor into a half-metal. This remarkable result demonstrates the relevance of many-body effects for spintronic materials.

The main features of the electronic structure of VAs [14] are shown schematically in Fig. 1. The $t_{2 g}$ states hybridize with the neighboring As $p$ states, forming wide bonding and antibonding hybrid bands. In contrast, the $e_{g}$ states form mainly nonbonding and narrow bands. The Fermi level falls between the $e_{g}$ and the antibonding $t_{2 g}$ in the majority-spin bands and between the bonding $t_{2 g}$ and the $e_{g}$ in the minority-spin bands. Thus, the material is a ferromagnetic semiconductor, showing a very narrow gap at $E_{F}$ (of the order of $50 \mathrm{meV}$ ) for majority spins and a much larger gap for minority spins. The spin moment, concentrated mainly at the $\mathrm{V}$ atoms, is an integer of exactly $M=2 \mu_{B}$ per unit formula, which is obvious by counting the occupied bands of the two spin directions. 
The exchange constants of VAs were calculated within GGA and adiabatic spin dynamics. The energy $E(\vec{q})$ of frozen magnons is calculated as a function of the wave vector $\vec{q}$ using the full-potential linearized augmented plane wave method [18], which allows one to evaluate the real-space exchange constants $J_{i j}$ by a Fourier transform of $E(\vec{q})$. The procedure is similar to the one used by Halilov et al. [19]. Using these exchange parameters in a Monte Carlo simulation of the corresponding classical Heisenberg Hamiltonian $E=-(1 / 2) \sum_{i j} J_{i j} \vec{M}_{i} \cdot \vec{M}_{j}$ (where $\vec{M}_{i}$ and $\vec{M}_{j}$ are the magnetic moments at sites $i$ and $j$ ), we obtain a Curie temperature $T_{C}=820 \mathrm{~K}$ by the fourth-order cumulant crossing point. This result agrees with the value of $T_{C}=830 \mathrm{~K}$ calculated in Ref. [20] using a similar method. The high Curie point is well above room temperature, making VAs a very promising candidate for applications in spintronics.

In order to take into account static correlations, we employ the GGA $+U$ method (using the values $U=$ $2 \mathrm{eV}, \quad J=0.9 \mathrm{eV}$ typical for $3 d$ transition metals $[21,22])$. Accordingly, we used the GGA equilibrium lattice parameter $a=5.69 \AA$ and a broadening $\delta$ of about $15 \mathrm{~K}$, which allows the majority-spin gap to be clearly resolved. For different lattice parameters (e.g., the InAs parameter), our local-density approximation (LDA) results agree with the ones previously reported $[14,20]$. The main difference between the GGA density of states (DOS) (see Fig. 2) and GGA $+U$ spectrum (not shown here) is, as expected, that within the GGA $+U$ the occupied, localized majority $e_{g}$ states are shifted to even lower energy, while the unoccupied, minority $e_{g}$ states are shifted to higher energy. The semiconducting character does not change, since the $e_{g}$ and $t_{2 g}$ bands remain separated for both spins; the majority-spin gap increases slightly but remains small.

In order to investigate dynamic correlation effects in VAs, we used a recently developed fully self-consistent in spin, charge, and self-energy LSDA + DMFT scheme [23]. This scheme uses for the LDA or GGA calculations the exact muffin-tin orbitals theory [24] and the full charge density technique. This method combines the accuracy of the full-potential method and the efficiency of the muffintin potential method [24]. Correlation effects are treated in the framework of dynamical-mean-field theory with a spinpolarized $T$-matrix fluctuation exchange type of quantum

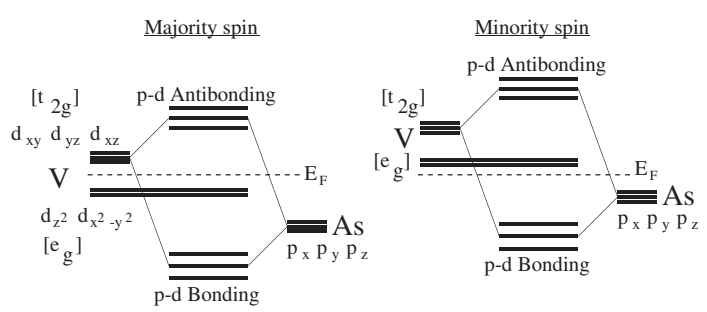

FIG. 1. Schematic representation of the $p$ - $d$ hybridization and bonding-antibonding splitting in VAs. impurity solver $[25,26]$. The computational details are described in Refs. [23,27,28].

In our calculations, we considered the standard representation of the zinc-blende structure with an fcc unit cell containing four atoms: $\mathrm{V}(0,0,0)$, As $(1 / 4,1 / 4,1 / 4)$, and two vacant sites at $(1 / 2,1 / 2,1 / 2)$ and $(3 / 4,3 / 4,3 / 4)$. The charge density is calculated by integrating the Green function along a complex contour up to the Fermi level. The multipole expansion of the charge density is cut off at $l_{\max }=8$, and the expansion of the Green function is cut off at $l_{\max }=3$. The convergence of the calculations was checked up to a number of $1505 k$ vectors and several sets between 22 and 30 complex energy points. The PerdewBurke-Ernzerhof [29] parametrization of the GGA exchange correlation potential was used. The one-particle, LSDA or GGA + DMFT Green function $G_{\sigma}(\vec{k} ; E)$ is related to the LSDA or GGA Green function $G_{\sigma}^{0}(\vec{k} ; E)=$ $\left[E+\mu-H_{\sigma}^{0}(\vec{k})\right]^{-1}$ and to the local (on-site) self-energy $\Sigma_{\sigma}(E)$ via the Dyson equation

$$
G_{\sigma}^{-1}(\vec{k} ; E)=E+\mu-H_{\sigma}^{0}(\vec{k})-\Sigma_{\sigma}(E)
$$

$H_{\sigma}^{0}(\vec{k})$ is the LSDA or GGA Hamiltonian, dependent on the Bloch vector $\vec{k}$, and the spin index $\sigma \in\{\uparrow, \downarrow\}$. $\mu$ is the chemical potential. The many-body effects beyond the LSDA or GGA are described by the multiorbital interacting Hamiltonian $\frac{1}{2} \sum_{i\{m, \sigma\}} U_{m m^{\prime} m^{\prime \prime} m^{\prime \prime \prime}} c_{i m \sigma}^{\dagger} c_{i m^{\prime} \sigma^{\prime}}^{\dagger} c_{i m^{\prime \prime \prime} \sigma^{\prime}} c_{i m^{\prime \prime} \sigma}$, where $m$ are local orbitals at site $i ; c^{\dagger}$ and $c$ denote creation and destruction operators, respectively. For the multiorbital Hamiltonian, the on-site Coulomb interactions are expressed in terms of two parameters $U$ and $J$ [7]. In order to avoid "double counting," the static part of the selfenergy is subtracted; i.e., $\Sigma_{\sigma}(E)$ is replaced with $\Sigma_{\sigma}(E)-$ $\Sigma_{\sigma}(0)$, as $\Sigma_{\sigma}(0)$ is already included in the LSDA or GGA part of the Green's function. It has been proven that this type of "metallic" double counting is suitable for medium correlated $d$-electron systems [30].

The computational results for the GGA and GGA + DMFT densities of states are presented in Fig. 2. The nonquasiparticle (NQP) states in the minority-spin band are visible just above the Fermi level (inset), predicted also by previous calculations $[27,28]$. The weak spectral weight of the NQP state is due to the fact that the Fermi level is close to the right edge of the minority-spin gap, as discussed for CrAs having a similar structure [28]. The origin of the NQP states is connected to the "spin-polaron" processes: The spin-down low-energy electron excitations, forbidden for the HMF in the one-particle picture, turn out to be possible as superpositions of spin-up electron excitations and virtual magnons [8,9]. The local spin moments at the $\mathrm{V}$ atoms do not change significantly (less than 5\%).

However, in the case of VAs, another correlation effect appears: The small majority-spin gap at $E_{F}$ closes, making the material half-metallic. This prediction is the central result of our work. 


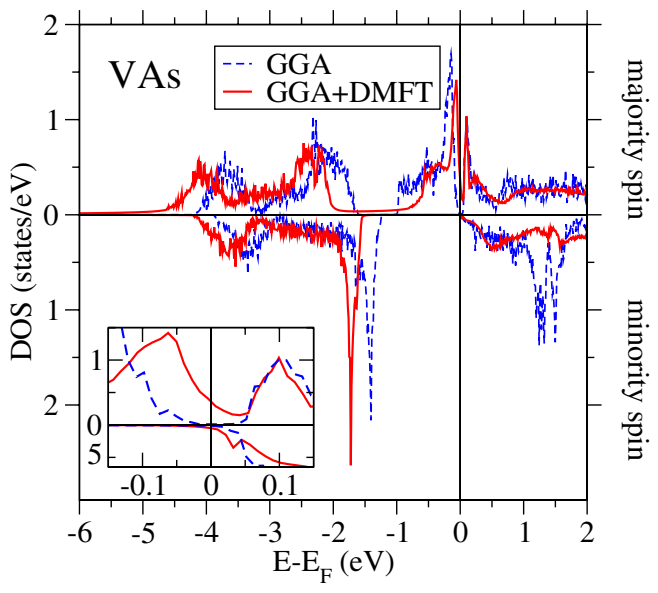

FIG. 2 (color online). DOS of VAs within the GGA (dashed blue line) and GGA + DMFT (solid red line) for a temperature of $T=200 \mathrm{~K}, U=2 \mathrm{eV}$, and $J=0.9 \mathrm{eV}$. Inset: Focus around $E_{F}$ showing the semiconducting gap within the GGA. To illustrate the minority-spin NQP states, a 10 times larger scale for the spin-down channel is used.

In order to investigate the mechanism of the gap closure for the majority-spin channel, we look at the behavior of the electron self-energy. The quadratic form of the imaginary part of the majority-spin self-energy, $\operatorname{Im} \Sigma_{\uparrow}(E) \sim(E-$ $\left.E_{F}\right)^{2}$, visible in both Figs. 3 and 4 , indicates a Fermi-liquid behavior, as opposed to $\Sigma_{l}(E)$, which shows a suppression around $E_{F}$ due to the band gap, as well as a peculiar behavior for $E>E_{F}$ related to the existence of NQP states $[8,9]$.

From the Dyson equation (1), one can see that the real part of the self-energy $\operatorname{Re} \Sigma_{\sigma}(E)$ causes a shift of the LDA energy levels. Therefore, due to the nonzero $\Sigma_{\uparrow}^{e_{g}}$, the $e_{g}$ orbitals in the close vicinity of the Fermi level are pushed closer to $E_{F}$. This renormalization of the levels is connected to the large value of $\operatorname{Re}(\partial \Sigma / \partial E)_{E_{F}}<0$, pushing states in the vicinity of $E_{F}$ more closer to $E_{F}$. This causes occupied levels to be renormalized to higher energy and unoccupied levels to lower energy. Notice that this effect is completely opposite to the GGA $+U$ results, discussed above. In addition to this shifting, the $e_{g}$ peak is broadened by correlations, its tail reaching over the Fermi level (Fig. 2, inset). Thus, our finite-temperature GGA + DMFT calculations demonstrate the closure of the narrow gap in the spin-up channel, which is produced by the correlation-induced Fermi-liquid renormalization and spectral broadening. At the same time, NQP states appear for the minority-spin channel just above $E_{F}$.

The slope of the majority-spin channel self-energy is almost a constant as a function of temperature: $\operatorname{Re}\left(\partial \Sigma_{\uparrow} / \partial E\right)_{E_{F}} \approx-0.4$ between 200 and $500 \mathrm{~K}$. The physical content of this lies in the quasiparticle weight $Z=$ $\left(1-\partial \operatorname{Re} \Sigma_{\uparrow} / \partial E\right)^{-1}$, measuring the overlap of the quasiparticle wave function with the original one-electron wave function, having the same quantum numbers. Our numerical results indicate that $Z \approx 0.7$ is quite temperature independent for small temperatures. Thus, we could expect this renormalization to hold down to zero temperature. As a consequence, the closure of the gap in the majority channel is a quantum effect, originating from the multiorbital nature of the local Coulomb interaction (orbitals are squeezed towards $E_{F}$ ) rather than an effect of temperature. We have verified that a similar gap closure is obtained for larger values of $U$, namely, $U=4$ and $6 \mathrm{eV}$, although the latter values should be taken with some caution in our fluctuation exchange calculation, which is, in principle, appropriate only in weak to intermediate coupling. As a general tendency, increasing $U-J$ produces a stronger Fermi-liquid renormalization in the majority-spin channel. We have also verified that the same effect is evidenced for $J=0 \mathrm{eV}$.

Recent mean-field (LDA $+U$ ) calculations by Anisimov et al. [31] yield a first-order, semiconductor to ferromagnetic metal transition as a function of doping in the $\mathrm{FeSi}_{1-x} \mathrm{Ge}_{x}$ alloy. In contrast, our calculation clearly shows that in VAs the closure of the semiconducting gap for majority spins cannot be captured by a static approach; dynamic correlation contributions in the multiorbital model are required.
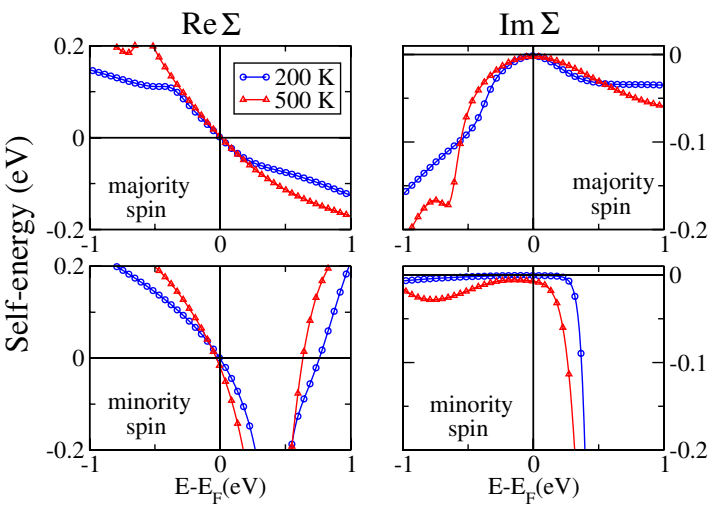

FIG. 4 (color online). Energy dependence of real and imaginary parts of the self-energy $\Sigma_{\sigma}(E)$ for the $e_{g}$ orbitals. 
In summary, we have investigated the electronic structure and correlation effects in the zinc-blende alloy VAs. On the one hand, our density-functional theory calculations within the GGA predict this material to be a ferromagnetic semiconductor with a tiny gap of about $50 \mathrm{meV}$ in the majority-spin DOS. On the other hand, dynamical effects described by LDA + DMFT destroy the narrow band gap and turn the material into a half-metallic ferromagnet. According to our results, the closure of the band gap is due to the multiorbital nature of the local Coulomb interaction and can be described as a strong correlationinduced Fermi-liquid-like renormalization of majorityspin states accompanied by a lifetime broadening. At the same time, in the minority-spin channel nonquasiparticle states appear just above $E_{F}$. We stress that these results are a consequence of the interaction of spin-up electrons with spin-flip excitations. Neither the closure of the gap nor the nonquasiparticle states can be obtained in the static LDA $+U$ approximation. Our LDA or GGA calculation supplemented by a Monte Carlo simulation also predicts a high Curie temperature of $820 \mathrm{~K}$, which makes this material of interest for technological applications. We expect $T_{c}$ to be not much affected by dynamical correlation, for the same reason for which the effective exchange interaction parameters are not affected, as was demonstrated in recent works [25,32].

The revealed half-metallic (instead of semiconducting) behavior has important consequences in the potential applications of VAs in spintronics. In contrast to allsemiconductor-based spin-injection devices [3], which avoid the resistivity mismatch problem, half-metals can be applied in giant magnetoresistance or, if interface states are eliminated [33], in tunneling magnetoresistance. As our calculations show, in the prediction of new spintronic materials, correlation effects play a decisive role. While in some materials these are detrimental for half-metallicity due to the introduction of spectrum in the minority-spin gap [27], the present case is an example in which correlations turn out to be favorable for a high spin polarization. The metallic nature of the majority-spin channel would be visible in resistivity measurements. Therefore, the experimental realization of zinc-blende VAs would provide a test of our prediction. Further research should address the issue of the stability of the half-metallic ferromagnetic state in a zinc-blende structure. Some work in this direction has been already carried out $[34,35]$. DMFT total-energy calculations along the lines described in Ref. [36] are in progress.

We thank Dr. R. A. de Groot for helpful discussions. We acknowledge financial support by the Research Center Jülich (L. C.) and by the FWF Project No. P18505-N16 (L. C. and E. A.).

[1] R. A. de Groot et al., Phys. Rev. Lett. 50, 2024 (1983).
[2] V. Yu. Irkhin and M.I. Katsnelson, Phys. Usp. 37, 659 (1994).

[3] I. Žutic̀ et al., Rev. Mod. Phys. 76, 323 (2004).

[4] I. Turek et al., Psi-k Scientific Highlight (Oct. 2003), available at http://psi-k.dl.ac.uk; E. Sasioglu et al., J. Phys. Condens. Matter 17, 3915 (2005).

[5] M. Ležaić et al., cond-mat/0512277.

[6] V. I. Anisimov et al., J. Phys. Condens. Matter 9, 7359 (1997).

[7] A. I. Lichtenstein and M. I. Katsnelson, Phys. Rev. B 57, 6884 (1998); M. I. Katsnelson and A. I. Lichtenstein, J. Phys. Condens. Matter 11, 1037 (1999).

[8] D. M. Edwards and J. A. Hertz, J. Phys. F 3, 2191 (1973).

[9] V. Yu. Irkhin and M. I. Katsnelson, Sov. Phys. Solid State 25, 1947 (1983).

[10] V. Yu. Irkhin and M. I. Katsnelson, Eur. Phys. J. B 30, 481 (2002).

[11] E. McCann and V.I. Fal'ko, Phys. Rev. B 68, 172404 (2003).

[12] E. L. Nagaev, Physics of Magnetic Semiconductors (Mir, Moscow, 1983).

[13] H. Ohno, Science 281, 951 (1998); J. Magn. Magn. Mater. 200, 110 (1999).

[14] I. Galanakis and P. Mavropoulos, Phys. Rev. B 67, 104417 (2003).

[15] H. Akinaga et al., Jpn. J. Appl. Phys. 39, L1118 (2000); M. Mizuguchi et al., J. Magn. Magn. Mater. 239, 269 (2002).

[16] J. H. Zhao et al., Appl. Phys. Lett. 79, 2776 (2001).

[17] J. Okabayashi et al., Phys. Rev. B 70, 233305 (2004).

[18] http://www.flapw.de.

[19] S. V. Halilov et al., Phys. Rev. B 58, 293 (1998).

[20] B. Sanyal, L. Bergqvist, and O. Eriksson, Phys. Rev. B 68, 054417 (2003).

[21] G. Kotliar and D. Vollhardt, Phys. Today 57, No. 3, 53 (2004); G. Kotliar et al., cond-mat/0511085.

[22] F. Aryasetiawan et al., cond-mat/0603138.

[23] L. Chioncel et al., Phys. Rev. B 67, 235106 (2003).

[24] O. K. Andersen et al., Phys. Rev. B 62, R16 219 (2000); L. Vitos, Phys. Rev. B 64, 014107 (2001); L. Vitos et al., Comput. Mater. Sci. 18, 24 (2000).

[25] M. I. Katsnelson and A. I. Lichtenstein, Eur. Phys. J. B 30, 9 (2002).

[26] L. V. Pourovskii et al., Phys. Rev. B 72, 115106 (2005).

[27] L. Chioncel et al., Phys. Rev. B 68, 144425 (2003); L. Chioncel et al., Phys. Rev. Lett. 96, 137203 (2006).

[28] L. Chioncel et al., Phys. Rev. B 71, 085111 (2005).

[29] J. P. Perdew et al., Phys. Rev. Lett. 77, 3865 (1996).

[30] A. G. Petukhov et al., Phys. Rev. B 67, 153106 (2003); A. I. Lichtenstein et al., Phys. Rev. Lett. 87, 067205 (2001).

[31] V. I. Anisimov et al., Phys. Rev. Lett. 89, 257203 (2002).

[32] M. I. Katsnelson and A. I. Lichtenstein, Phys. Rev. B 61, 8906 (2000).

[33] Ph. Mavropoulos et al., Phys. Rev. B 72, 174428 (2005).

[34] M. Shirai, Physica (Amsterdam) 10E, 143 (2001); J. Appl. Phys. 93, 6844 (2003).

[35] W. H. Xie et al., Phys. Rev. Lett. 91, 037204 (2003).

[36] Y. J. Zao and A. Zunger, Phys. Rev. B 71, 132403 (2005). 